\title{
Inside the lungs of COVID-19 disease
}

\section{Diego Aguiar $^{1}$ (i) $\cdot$ Johannes Alexander Lobrinus ${ }^{2} \cdot$ Manuel Schibler $^{3,4} \cdot$ Tony Fracasso $^{1} \cdot$ Christelle Lardi $^{1}$}

Received: 3 April 2020 / Accepted: 19 May 2020/Published online: 26 May 2020

(C) Springer-Verlag GmbH Germany, part of Springer Nature 2020

\begin{abstract}
In the setting of the coronavirus disease 2019 (COVID-19) pandemic, only few data regarding lung pathology induced by severe acute respiratory syndrome coronavirus 2 (SARS-CoV-2) is available, especially without medical intervention interfering with the natural evolution of the disease. We present here the first case of forensic autopsy of a COVID-19 fatality occurring in a young woman, in the community. Diagnosis was made at necropsy and lung histology showed diffuse alveolar damage, edema, and interstitial pneumonia with a geographically heterogeneous pattern, mostly affecting the central part of the lungs. This death related to COVID-19 pathology highlights the heterogeneity and severity of central lung lesions after natural evolution of the disease.
\end{abstract}

Keywords SARS-CoV-2 $\cdot$ COVID-19 $\cdot$ Post-mortem $\cdot$ Diffuse alveolar damage $\cdot$ Interstitial pneumonia

\section{Introduction}

In late 2019, a novel human coronavirus, later named severe acute respiratory syndrome coronavirus 2 (SARS-CoV-2), was discovered in Wuhan (China), in cases of fatal pneumonia [1]. Coronaviruses are known to induce a wide range of pathology ranging from common cold to severe acute respiratory syndrome. Most of the viruses present in the family of Coronaviridae are known to induce primarily self-limited upper respiratory diseases with the exceptions of two Betacoronaviruses, namely SARS-CoV and MERS-CoV. The former was responsible for a pandemic of viral pneumonia which began in the Guangdong province (China) in late 2002 and peaked globally in the early 2003 . The latter was discovered in 2012 and was responsible for cases of fatal viral

This article is part of the Topical Collection on COVID-19

Diego Aguiar

diego.aguiar@hcuge.ch

1 University Center of Legal Medicine (CURML), Geneva University Hospital, Geneva, Switzerland

2 Department of Pathology, Geneva University Hospital, Geneva, Switzerland

3 Laboratory of Virology, Geneva University Hospital, Geneva, Switzerland

4 Infectious Diseases Division, Geneva University Hospital, Geneva, Switzerland pneumonia mainly in the Arabic peninsula, especially in Saudi Arabia. In late December 2019, SARS-CoV-2 was related to cases of fatal viral pneumonia. Since then, the virus rapidly spread worldwide and caused a new pandemic. To date, only limited data is available regarding the lung pathology induced by SARS-CoV-2. Forensic investigations continue to be necessary during COVID-19 pandemic when the manner of death is violent or undetermined. In mid-March 2020, our Center was confronted for the first time to the case of a potentially infected young woman who collapsed at home during confinement. No specific diagnosis was made before death. We present here the results of an exceptional case of forensic autopsy, showing extremely severe lung lesions of a COVID-19 disease diagnosed on post-mortem samples. This is to our knowledge, the first forensic autopsy performed on a young woman who died at home during confinement and was later diagnosed with COVID-19 disease.

\section{Case presentation}

A 31-year-old woman was found unconscious in her flat during the COVID-19 pandemic. Antitussive opioid sirup and ibuprofen were found at death scene. Paramedics called on site performed $30 \mathrm{~min}$ of unsuccessful resuscitation maneuvers before the patient was pronounced dead. The police was called because of the young age of the victim. According to preliminary police investigation, the woman was still alive 
approximatively two and a half hours before her body was found unconscious. She suffered from morbid obesity. Seven days prior to death, she started presenting cough. She attended medical attention twice in the previous days and was confined at home since then. The deceased worked as a cashier in a department store.

During the death scene investigation performed by the forensic pathologist, a rectal temperature of $41.4{ }^{\circ} \mathrm{C}$ was measured $2 \mathrm{~h}$ after death. A medico-legal autopsy was ordered by the public prosecutor and was performed the day after at the University Center of Legal Medicine in Geneva, Switzerland. Available recommendations for autopsy practice in suspected cases of COVID-19 were applied [2]. Practically, postmortem examination was performed in an autopsy room specially designed to perform investigations on infectious cases with highly renewable positive-pressure ventilation and down-draft tables. Autopsy staff was reduced to a minimum (resident, supervisor, and technician) with transfer airlock adjacent to the autopsy room. The staff was adequately trained and wore appropriate personnel protective equipment (surgical scrub suit, surgical hat, eye protection glasses, FFP3 mask, waterproof gown including forearms, rubber boots, and cutresistant gloves).

Post-mortem computed tomography (PMCT) revealed diffuse, bilateral ground-glass opacities associated with panlobar air space consolidations, and air bronchograms.

Gross autopsy findings included morbid obesity (height $167 \mathrm{~cm}$; weight $170.7 \mathrm{~kg}$; body mass index of $61.2 \mathrm{~kg} / \mathrm{m}^{2}$ ), heavy lungs grossly firm and rubbery $(1220 \mathrm{~g}$ right; $1000 \mathrm{~g}$ left) with hemorrhagic edema bilaterally, tracheobronchial and pleural effusion, petechiae of the skin, and signs of shock (petechial hemorrhages of the visceral and parietal serosa of the abdomen, variegated "nutmeg" liver appearance, cortical pallor with medullary congestion of the kidneys). Cut sections of the lungs revealed heterogeneous areas of whitish consolidation without any purulent discharge. Heart weight normalized for BMI was normal (460 g) with wall thickness of $1.3 \mathrm{~cm}$ at the left ventricle and interventricular septum and $0.3 \mathrm{~cm}$ at the right ventricle. Fatty streaks were noticed at the anterior interventricular branch of left coronary artery and carotid bifurcations. No signs of meningitis or encephalitis were found at gross examination or microscopic investigation.

Histology of the lungs showed alveolar damage with a geographically heterogeneous pattern, the central part of the lungs being mostly affected (Fig. 1a). Alveolar compartment showed mainly edema, diffuse alveolar damage (DAD) in his exudative phase with the presence of hyaline membranes in some alveoli (Fig. 1c), deposit of fibrin as cotton wools within the alveoli (Fig. 1b), and moderate type II pneumocytes hyperplasia which were mainly desquamated (Fig. 2a). In the alveolar exudate, there were also moderate amount of intraalveolar macrophages (Fig. 2c) and only scant polymorphonuclear neutrophils (PMN) and lymphocytes. Focal areas of intraalveaolar hemorrhage and bacterial proliferation were also present. No viral inclusions or giant multinucleated cells were noted. Interstitial compartment showed edema, vascular stasis, lympho-monocytic infiltrates (majority of $\mathrm{T}$ lymphocytes (CD3+, Fig. 2d) over monocytes and exempt of B lymphocytes (CD20+)), and abundant megakaryocytes. Within the alveolar septa and mainly into the capillaries, abundant PMN were also seen, indicating margination and diapedesis. No signs of increased collagen deposition within the alveoli or the intersitium were noticed (Masson trichrome stain). Bronchi and bronchioles showed only minimal lymphocytic infiltrates within their walls and did not display any sign of mucosal necrosis. Histological analysis of other organs showed mild chronic tracheitis and microabscesses in the liver parenchyma. At microscopy, we did not observe any signs of cardiac hypertrophy.

Post-mortem microbiological (bacterial and virological) analysis was performed. A lower respiratory tract (tracheobronchial) swab was performed and tested positive for SARS-CoV-2 by real-time reverse transcription PCR (rRTPCR), with a threshold cycle $(\mathrm{Ct})$ value of 21.4 (E gene, TibMolbiol assay). An rRT-PCR-based respiratory virus panel run on the same specimen, detecting influenza viruses A and B, respiratory syncytial virus $\mathrm{A}$ and $\mathrm{B}$, adenovirus, human metapneumovirus, rhinoviruses, bocavirus, other coronaviruses (OC43, NL63, 229E and HKU1), and parainfluenza viruses 14, was negative. Bacterial analysis performed on blood, lungs, liver, spleen, and cerebrospinal fluid revealed mixed flora. Postmortem biochemistry was indicative of an inflammatory process with elevated C-reactive protein of $48 \mathrm{mg} / \mathrm{l}$ (normal value $\leq 10 \mathrm{mg} / \mathrm{l})$, without an elevated procalcitonin value $(0.06 \mu \mathrm{g} / \mathrm{l})$. Dextromethorphan was found in blood screening.

At the end of the procedure, the body was placed in a hygienic sanitary bag according to standard internal procedures, applying additional specific contact avoiding measures. According to local recommendations edited by our local hygiene, infection control, and prevention team, cleanup techniques of the autopsy room did not differ from usual ones.

\section{Discussion}

SARS-CoV-2 is known to cause acute respiratory distress syndrome. To date, only few data regarding SARS-CoV-2related lung pathology are available [3-5] and mainly based on surgical material [5], post-mortem biopsies [4], or minimal invasive autopsy [3]. To our knowledge, we report here the first case of a forensic autopsy of a young COVID-19 fatality diagnosed at necropsy without any medical intervention interfering with the natural evolution of the disease. Lung pathology revealed DAD and interstitial pneumonia. The pattern of injury was geographically heterogeneous affecting primarily 


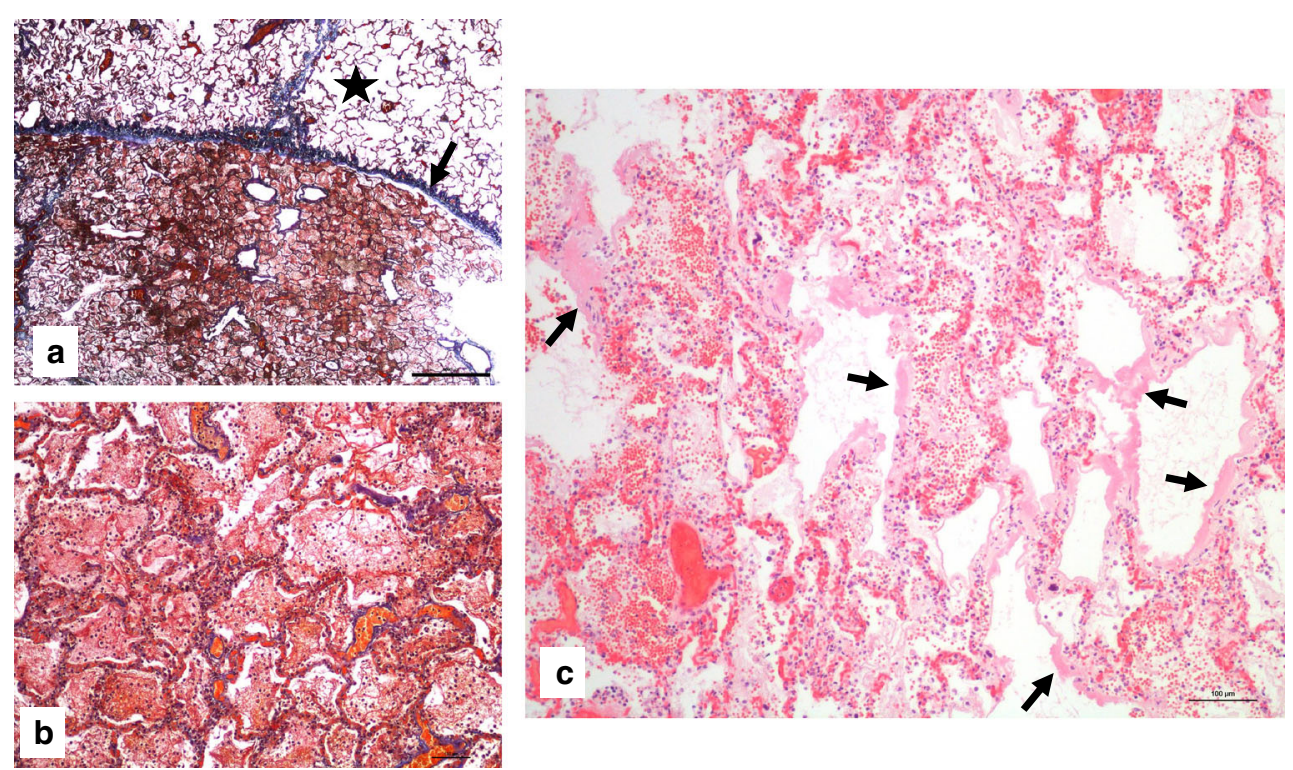

Fig. 1 a Upper right lobe lung parenchyma showing geographical heterogeneity of pulmonary lesions, with two samples of lung parenchyma in contact. The central sample is at the bottom presenting diffuse filling of the alveolar space with fibrin and the peripheral sample is at the top (black star) with clear open alveolar space. Arrow indicate visceral pleura (acid fuchsin-orange $\mathrm{G}$ stain $(\mathrm{SFOG}), \times 20)$. b Magnification of diffuse filling of the alveolar space with fibrin (acid fuchsin-orange $\mathrm{G}$ stain $(\mathrm{SFOG}), \times 100$ ). c Hyaline membranes (black arrows) indicating diffuse alveolar damage (hematoxylin and eosin, $\times$ 100) central parts of the lungs and preserving the peripheral parts. An early phase of secondary bacterial infection was noticed within the alveoli, with margination of PMNs. Abundant megakaryocytes can also be explained by the early stage of infection, reflecting release of hemopoietic cells by the bone marrow. However, according to the post-mortem biochemistry, death was attributed to pulmonary changes related to SARS-CoV-2 and high fever without implication of a secondary bacterial infection (low procalcitonin value).

Overall, our findings are in agreement with those described in literature regarding SARS-CoV-2 infection [4]. Some features were however different, such as the absence of
Fig. 2 a Moderate type II pneumocytes hyperplasia (black arrows) with some desquamation (pankeratin, $\times 200$ ). b Unaffected area of lung parenchyma with normal pneumocytes I and II morphology (pankeratin, $\times 200$ ). c Moderate intra-alveolar macrophages $(\mathrm{CD} 68, \times 200)$. d Moderate amount of $\mathrm{T}$ lymphocytes infiltration in the interstitium $(\mathrm{CD} 3, \times 100)$
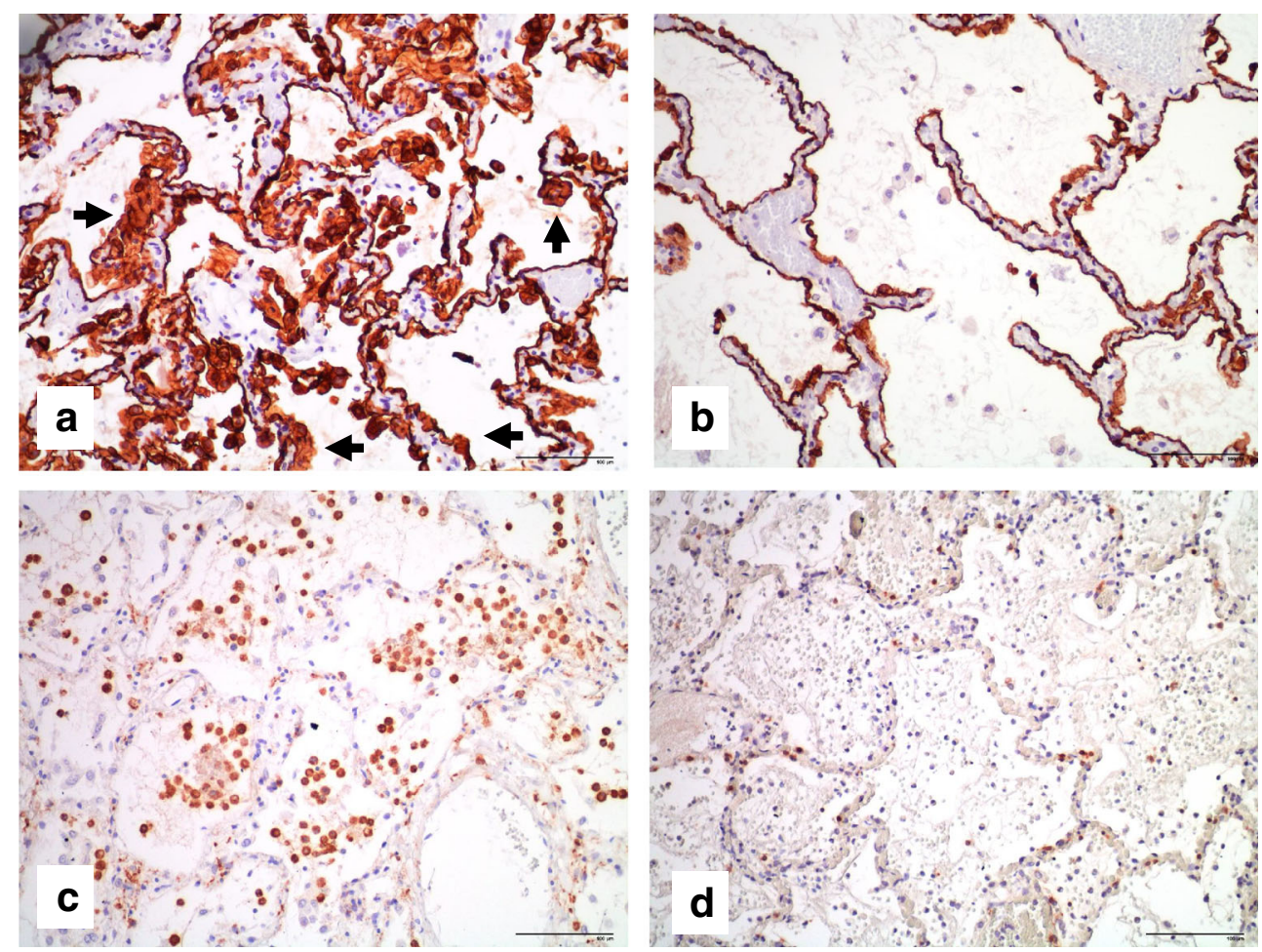
multinucleated syncytial cells [3-5], fibroblastic plugs [5], or hyaline thrombi in microvessels [3]. The lesions induced by SARS-CoV-2 were similar to those caused by other members of the Coronaviridae family, especially the Betacoronaviruses SARS-CoV [6, 7] and MERS-CoV [8]. One particular element we observed was the complete absence of B lymphocytes within the interstitial compartment despite the lymphomonocytic infiltrate. This finding is in line with the idea that the immune response in the lung parenchyma is mainly mediated by T lymphocytes [9]. However, whether this feature is also present in other cases of SARS-CoV-2 infection or in other types of Betacoronaviruses lung infections remains unclear.

To date, several modes of transmission of the SARS-CoV2 virus have been proposed such as respiratory droplets, contact routes, airborne transmission, and theoretically by oralfecal transmission. In the present case, because of the professional activity of the victim (cashier in a department store), the presumed mode of transmission was by respiratory droplets or contact based.

We recommend that forensic institutes performing autopsies on suspected COVID-19 cases follow appropriate guidelines for this type of procedure [2]. As such, autopsy should be performed preferably in an infectious diseases dedicated and equipped room, with staff kept to a minimum, adequately trained, and geared with personnel protective equipment.

This community death of a young woman related to COVID-19 pathology highlights the heterogeneity and severity of central lung lesions after natural evolution of the disease in conditions of confinement.

\section{Compliance with ethical standards}

Conflict of interest The authors declare that they have no conflict of interest.
Informed consent Written consent for publication was provided by the public prosecutor according to local agreement.

\section{References}

1. Zhu N, Zhang D, Wang W, Li X, Yang B, Song J, Zhao X, Huang B, Shi W, Lu R, Niu P, Zhan F, Ma X, Wang D, Xu W, Wu G, Gao GF, Tan W, China Novel Coronavirus Investigating and Research Team (2020) A novel coronavirus from patients with pneumonia in China. N Engl J Med 382(8):727-733

2. Osborn M, Lucas S, Stewart R et al. (2020) Autopsy practice relating to possible cases of COVID-19 (2019-nCov, novel coronavirus from China 2019/2020). R Coll Pathol

3. Yao XH, Li TY, He ZC et al (2020) A pathological report of three COVID-19 cases by minimally invasive autopsies. Zhonghua Bing Li Xue Za Zhi 49(0):E009. https://doi.org/10.3760/cma.j.cn11215120200312-00193

4. Xu Z, Shi L, Wang Y et al (2020) Pathological findings of COVID19 associated with acute respiratory distress syndrome. Lancet Respir Med S2213-2600(20):30076-3007X. https://doi.org/10. 1016/S2213-2600(20)30076-X

5. Tian S, Hu W, Niu L, Liu H, Xu H, Xiao S-J (2020) Pulmonary pathology of early-phase 2019 novel coronavirus (COVID-19) pneumonia in two patients with lung cancer. J Thorac Oncol S15560864(20):30132-30135. https://doi.org/10.1016/j.jtho.2020.02.010

6. Hwang DM, Chamberlain DW, Poutanen SM, Low DE, Asa SL, Butany J (2005) Pulmonary pathology of severe acute respiratory syndrome in Toronto. Mod Pathol 18(1):1-10. https://doi.org/10. 1038/modpathol.3800247

7. Lang Z-W, Zhang L-J, Zhang S-J, Meng X, Li JQ, Song CZ, Sun L, Zhou YS, Dwyer DE (2003) A clinicopathological study of three cases of severe acute respiratory syndrome (SARS). Pathology 35(6):526-531

8. Ng DL, Hosani FA, Keating MK et al (2016) Clinicopathologic, immunohistochemical, and ultrastructural findings of a fatal case of Middle East respiratory syndrome coronavirus infection in the United Arab Emirates, April 2014. Am J Pathol 186(3):652-658

9. Chen K, Kolls JK (2013) T cell-mediated host immune defenses in the lung. Annu Rev Immunol 31:605-633

Publisher's note Springer Nature remains neutral with regard to jurisdictional claims in published maps and institutional affiliations. 